\title{
Efecto de dos colectas de semen en una temporada reproductiva sobre la calidad seminal de cachama blanca (Piaractus brachypomus)
}

\author{
Effect of two semen collection in a breeding season on the seminal quality of \\ red-bellied pacu (Piaractus brachypomus)
}

\author{
Roger Oswaldo Suárez Martínezi,2,3,4, Víctor Mauricio Medina Robles', \\ Pablo Emilio Cruz Casallas ${ }^{1}$
}

\section{Resumen}

\begin{abstract}
Se evaluó el efecto de dos colectas de semen realizadas en la misma temporada reproductiva sobre los parámetros espermáticos y composición fisicoquímica del plasma seminal de Piaractus brachypomus. El semen de nueve machos inducidos hormonalmente con extracto hipofisiario de carpa (EHC) fue colectado en abril y mayo de 2014. Se estudiaron los cambios entre colectas del volumen seminal (VS), movilidad espermática (ME), tiempo de activación (TA), concentración espermática (CE) y variables fisicoquímicas $\left(\mathrm{Na}^{+}, \mathrm{Ca}^{2+}, \mathrm{Mg}^{2+}, \mathrm{Cl}^{-}, \mathrm{K}^{+}\right.$, glucosa, triglicéridos, proteína, osmolaridad y $\left.\mathrm{pH}\right)$. Se observaron diferencias significativas entre colectas para $\mathrm{Na}^{+}$, triglicéridos y osmolaridad $(\mathrm{p}<0.05)$ $\mathrm{y} \mathrm{Ca}^{2+}(\mathrm{p}<0.01)$. Por otro lado, no se encontraron diferencias significativas en VS, ME, TA y CE entre colectas. La colección de semen de un reproductor inducido hormonalmente en dos oportunidades dentro de una misma temporada reproductiva en es una alternativa de manejo viable en $P$. brachypomus, aunque los componentes plasmáticos pueden variar por efecto del muestreo.
\end{abstract}

Palabras clave: calidad seminal; bioquímica; iones; fertilidad

${ }^{1}$ Grupo de Investigación sobre Reproducción y Toxicología de Organismos Acuáticos (GRITOX), Instituto de Acuicultura de los Llanos (IALL), Facultad de Ciencias Agropecuarias y Recursos Naturales, Universidad de los Llanos, Villavicencio, Meta, Colombia

${ }^{2}$ Grupo de Investigación en Manejo y Producción de Especies con Potencial Zootécnico (GRIPEPZ), Programa de Zootecnia, Facultad de Ciencias Agropecuarias, Universidad de Cundinamarca, Fusagasugá, Cundinamarca, Colombia

${ }^{3}$ Grupo de Investigación Ciencia Animal, Programa de Medicina Veterinaria y Zootecnia, Facultad de Ciencias Agropecuarias, Universidad de Ciencias Aplicadas y Ambientales (U.D.C.A.), Bogotá, Colombia

${ }^{4}$ E-mail: rosuarez@udca.edu.co

Proyecto financiado y ejecutado con recursos de la Convocatoria para Proyectos de Investigación, Desarrollo Tecnológico e Innovación - 2013, Dir. General de Investigaciones - UNILLANOS y II Convocatoria Interna para financiar proyectos de investigación - 2012 - UCundinamarca

Recibido: 29 de noviembre de 2018

Aceptado para publicación: 18 de junio de 2019 
The effect of two semen collections performed in the same breeding season on the sperm parameters and physicochemical composition of the seminal plasma of Piaractus brachypomus was evaluated. The semen of nine hormone-induced males with hypophysial carp extract (HCE) was collected in April and May 2014. Changes between semen collections in seminal volume (SV), sperm motility (SM), motility duration (MD), sperm concentration (SC) and biochemical composition $\left(\mathrm{Na}^{+}, \mathrm{Ca}^{2+}, \mathrm{Mg}^{2+}, \mathrm{Cl}^{-}, \mathrm{K}^{+}\right.$, glucose, triglycerides, protein, osmolarity and $\mathrm{pH}$ ) were evaluated. Significant differences were observed between collections for $\mathrm{Na}^{+}$, triglycerides and osmolarity $(\mathrm{p}<0.05)$ and $\mathrm{Ca}^{2+}$ $(\mathrm{p}<0.01)$. On the other hand, no significant differences were found in SV, SM, MD and SC between collections. The collection of semen from a hormonally induced male in two occasions in one reproductive season is a viable alternative for $P$. brachypomus, although plasma components may vary due to sampling.

Keywords: semen quality; biochemistry; ions; fertility

\section{INTRODUCCIÓN}

Piaractus brachypomus es un carácido propio de la Orinoquía y de la Amazonia, destacado por ser la especie nativa de mayor producción en Colombia, alcanzando 22455 toneladas/año (MADR, 2018). En la Orinoquía, esta especie inicia su maduración y reproducción al inicio de la estación lluvio$\mathrm{sa}$, esto es a fines de marzo hasta mediados de junio (Cruz-Casallas et al., 2011). A pesar del uso de inductores hormonales para reproducción en cautiverio (Fresneda et al., 2004), la producción de alevinos se encuentra en parte limitada por la ausencia de gametos de machos durante gran parte del año.

La calidad espermática ha sido definida como la capacidad de un espermatozoide para fertilizar un oocito con el subsecuente desarrollo de un embrión normal (Bobe y Labbé, 2010), y depende de características celulares, así como de los constituyentes del plasma seminal (Migaud et al., 2013). El plasma seminal está constituido a partir de secreciones testiculares originadas al interior del sistema de ductos espermáticos, donde además, se almacenan espermatozoides provenientes de los túbulos seminíferos (Lahnsteiner y Patzner, 2009). Las condiciones proporcionadas al plasma seminal por las secreciones de los ductos confieren a los espermatozoides la inmovilidad que los caracteriza (Mochida et al., 1999; Alavi y Cosson, 2005; 2006, Rashid et al., 2018). La valoración espermática incluye parámetros como concentración, morfología y movilidad y en el plasma seminal, factores como osmolaridad, $\mathrm{pH}$, iones, enzimas, metabolitos, azúcares, vitaminas y lípidos entre otros compuestos (Rurangwa et al., 2004; Robles et al., 2008).

Los constituyentes minerales del plasma seminal son determinantes de la calidad espermática, su presencia o ausencia afectan la osmolaridad, el pH y la activación de la movilidad (Morisawa et al., 1983a,b; Dzyuba y Cosson, 2014; Cejko et al., 2015). En la mayoría de las especies estudiadas predominan cinco iones: sodio $\left(\mathrm{Na}^{+}\right)$, potasio $\left(\mathrm{K}^{+}\right)$, cloruro $\left(\mathrm{Cl}^{-}\right)$, calcio $\left(\mathrm{Ca}^{2+}\right)$ y magnesio $\left(\mathrm{Mg}^{2+}\right)$, donde la composición y concentración iónica pueden variar ampliamente dentro de la misma especie, haciendo compleja la interpretación de los resultados obtenidos (Bozkurt et al., 2008, 2011; Alavi et al., 2009). 
Ante la posible escasez de machos, una opción que se puede considerar es la reutilización de parentales en la misma temporada reproductiva. El almacenamiento de espermatozoides viejos al interior de los testículos y la espermatogénesis continua ha sido demostrado en peces reofílicos sudamericanos (Batlouni et al., 2006; De Souza et al., 2015). Por otro lado, Kuradomi et al. (2016) ha demostrado que se puede colectar repetidamente machos $P$. mesopotamicus como método de obtención de semen sin afectar la calidad espermática.

El propósito de la presente investigación fue determinar el efecto de dos colectas en una misma temporada reproductiva sobre los parámetros espermáticos (volumen seminal, movilidad espermática, tiempo de activación, concentración espermática), y composición fisicoquímica del plasma seminal (concentración iónica, triglicéridos, glucosa, $\mathrm{pH}$, osmolaridad) del semen de cachama blanca Piaractus brachypomus.

\section{Materiales y Métodos}

El estudio se realizó en el Laboratorio de Reproducción de Peces Tropicales de la Estación Piscícola del Instituto de Acuicultura de los Llanos (IALL), Universidad de Los Llanos, en Villavicencio, Meta, Colombia. Se utilizaron nueve machos Piaractus brachypomus maduros con evidencia de la liberación de semen a través de la papila genital. Los ejemplares fueros trasladados desde estanques de tierra hacia piletas de concreto provistas de aireación y recambio continuo de agua. Se les identificó con marcas plásticas de colores en la aleta dorsal y se les indujo hormonalmente vía intramuscular con $4 \mathrm{mg} / \mathrm{kg}$ de extracto hipofisario de carpa (EHC) (Stoller Fisheries, USA). A las 18 horas de la inducción se les inyectó $300 \mathrm{mg} / \mathrm{l}$ de 2-fenoxietanol (Sigma) como tranquilizante y el semen fue colectado directamente en un tubo de ensayo graduado en mililitros, evitando contacto con agua, he- ces y orina. Este procedimiento se repitió en cada colecta (10/abril/2014 y 09/mayo/2014).

La concentración espermática fue evaluada a través de dos métodos: el espermatocrito (Hickman, 1958), calculado a partir de la relación porcentual entre el volumen de espermatozoides (spz) empaquetados respecto al volumen total de semen, luego de centrifugar sub-muestras individuales almacenadas en capilares (14000 g x $15 \mathrm{~min}$; EBBA 12, Hettich, Alemania); y el conteo espermático, que se realizó microscópicamente (40X) en muestras diluidas (1:1200) con solución salina $(\mathrm{NaCl} 0.9 \%)$, montadas en cámara de Neubauer. Cada muestra se evaluó por triplicado y el promedio de lecturas fue empleado para análisis subsecuentes.

La movilidad masal (\%) y el tiempo de activación (duración de la movilidad) se determinaron activando $20 \mu \mathrm{l}$ de semen con 180 $\mu 1$ de agua destilada sobre una lámina excavada (1.0-1.2 mm de profundidad, Micro Slides Premiere ${ }^{\circledR}$, China). El registro de cuatro cuadrantes se promedió tomándose como valor final de movilidad. El tiempo de activación se registró en segundos, inmediatamente después de la activación y hasta que la movilidad se redujo a $5 \%$.

La composición del plasma seminal fue determinada a partir de la centrifugación refrigerada de $1 \mathrm{ml}$ de semen (3000 g x $10 \mathrm{~min}$., $4{ }^{\circ} \mathrm{C}$; Eppendorf ${ }^{\circledR}, 5424 \mathrm{R}$, Alemania). El plasma sobrenadante fue almacenado a $-20^{\circ} \mathrm{C}$ en viales plásticos (Cruz-Casallas et al., 2007). Se determinó: 1) osmolaridad (osmometría de presión de vapor, VAPRO ${ }^{\circledR}$, 5600, USA); 2) pH (método potenciométrico, Hanna, HI-5221-01, España); 3) concentración de proteína (método biuret), glucosa (método enzimático glucosa-oxidasaperoxidasa) y triglicéridos (método glicerol fosfato-oxidasa-peroxidasa), (espectrofotometría VIS, BioSystems ${ }^{\circledR}$ BTS-350, España); y 4) concentración de iones $\mathrm{K}^{+}, \mathrm{Ca}^{2+}$, $\mathrm{Na}^{+}, \mathrm{Cl}^{-}, \mathrm{Mg}^{2+}$ (electrodo ión selectivo, Vitros, DT60II, USA). 
Cuadro 1. Características espermáticas del semen fresco de Piaractus brachypomus colectados en abril y mayo de 2014 ( $\mathrm{n}=9$ machos por mes)

\begin{tabular}{lcccc}
\hline \multirow{2}{*}{ Parámetro } & \multicolumn{2}{c}{ Abril } & \multicolumn{2}{c}{ Mayo } \\
\cline { 2 - 5 } & Media \pm s.d. & CV $(\%)$ & Media \pm s.d & CV (\%) \\
\hline Volumen seminal $(\mathrm{ml})$ & $7.44 \pm 2.18$ & 29.30 & $5.94 \pm 1.86$ & 31.31 \\
Movilidad espermática (\%) & $88.6 \pm 2.2$ & 2.5 & $87.4 \pm 3.5$ & 4.0 \\
Tiempo de activación (s) & $59.1 \pm 8.0$ & 13.6 & $60.7 \pm 10.1$ & 16.6 \\
Concentración espermática & $18.58 \pm 4.90$ & 26.37 & $15.81 \pm 5.26$ & 33.27 \\
(spz. 10\% $/ \mu 1)$ & & & & \\
Espermatocrito (\%) & $38.0 \pm 9.8$ & 25.7 & $36.3 \pm 11.5$ & 31.6 \\
\hline
\end{tabular}

Datos presentados como media \pm desviación estándar $(n=9)$

El efecto de dos colectas realizadas sobre la composición fisicoquímica del plasma seminal, así como de los parámetros espermáticos (volumen seminal, concentración espermática y tiempo de activación), fueron evaluadas a partir de una prueba t para muestras relacionadas, con una significancia del $5 \%(\dot{a}=0.05)$. El supuesto de distribución normal de los datos se verificó con la prueba de Shapiro-Wilk y la homocedasticidad con la prueba de Levene. Los datos se expresaron como media \pm desviación estándar (DS). Los análisis fueron ejecutados con el programa $\mathrm{SAS}^{\circledR}$ University Edition. Todos los procedimientos fueron realizados de conformidad con las normas y procedimientos para el uso de animales de laboratorio, descritas por el Committee of Care and Use of Laboratory Animal Resources (NRC, 2011), y fueron aprobados por el Comité de Bioética de la Universidad de los Llanos.

\section{Resultados}

No se observaron diferencias significativas entre los datos de calidad espermática por cada colecta (Cuadro 1). El volumen seminal (VS) presentó valores mínimos de $3.7 \mathrm{ml}$ y máximos de $11 \mathrm{ml}$, mientras que la concentración espermática $(\mathrm{CE})$ varió en- tre 7.44 y 25.8 x $10^{6}$ espermatozoide/ $\mu 1$. La movilidad espermática (ME) varió entre 80 y $90 \%$ y el tiempo de activación (TA) fluctuó entre 44 y $77 \mathrm{~s}$.

Se presentaron diferencias estadísticas entre colectas al nivel de $\mathrm{p}<0.05$ en osmolaridad, $\mathrm{Na}^{+}$y triglicéridos (TG) y al nivel de $\mathrm{p}<0.01$ en concentración de $\mathrm{Ca}^{2+}$ (Cuadro 2). Entre los iones, el $\mathrm{Na}^{+}$fue el de mayor concentración en las dos colectas, segui-

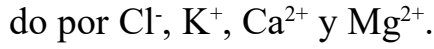

\section{Discusión}

La búsqueda de literatura destacó la escasa información publicada sobre las características plasmáticas seminales de $P$. brachypomus, la cual se limita a los reportes de Nascimento et al. (2010) y Sampaio Leite et al. (2014).

La ausencia de diferencias estadísticas entre colectas de los valores de VS, CE, ME, TA y espermatocrito presenta la posibilidad que un mismo macho sea colectado en dos ocasiones consecutivas sin alteración aparente de la calidad seminal. Los intervalos de tiempo entre colectas fueron de 30 días y coinciden con el estudio de Kuradomi et al. 
Cuadro 2. Composición bioquímica del plasma seminal de Piaractus brachypomus colectados en abril y mayo de 2014 ( $n=9$ machos por mes)

\begin{tabular}{|c|c|c|c|c|}
\hline \multirow[b]{2}{*}{ Parámetro } & \multicolumn{2}{|c|}{ Abril } & \multicolumn{2}{|l|}{ Mayo } \\
\hline & Media \pm s.d. & CV (\%) & Media \pm s.d. & CV (\%) \\
\hline $\mathrm{Ca}^{2+}(\mathrm{mmol} / \mathrm{l})$ & $3.45 \pm 1.34$ & 38.84 & $11.87 \pm 0.17 * *$ & 1.43 \\
\hline $\mathrm{Mg}^{2+}(\mathrm{mmol} / \mathrm{l})$ & $0.89 \pm 0.14$ & 15.73 & $1.01 \pm 0.16$ & 15.84 \\
\hline $\mathrm{Cl}^{-}(\mathrm{mmol} / \mathrm{l})$ & $119.00 \pm 8.03$ & 6.75 & $125.44 \pm 3.43$ & 2.73 \\
\hline $\mathrm{K}^{+}(\mathrm{mmol} / \mathrm{l})$ & $38.11 \pm 4.67$ & 12.25 & $37.22 \pm 5.26$ & 14.13 \\
\hline $\mathrm{Na}^{+}(\mathrm{mmol} / \mathrm{l})$ & $129.89 \pm 3.55$ & 2.73 & $134.78 \pm 3.53^{*}$ & 2.62 \\
\hline Glucosa (mg/dl) & $20.11 \pm 13.48$ & 67.03 & $18.44 \pm 13.22$ & 71.69 \\
\hline Triglicéridos (mg/dl) & $17.56 \pm 2.92$ & 16.63 & $21.11 \pm 2.47^{*}$ & 11.70 \\
\hline Proteínas (mg/dl) & $41.67 \pm 20.81$ & 49.94 & $30.56 \pm 14.17$ & 46.36 \\
\hline $\mathrm{pH}$ & $8.11 \pm 0.32$ & 3.95 & $7.98 \pm 0.20$ & 2.51 \\
\hline Osmol (mOsmol/kg) & $261.53 \pm 19.89$ & 7.61 & $307.55 \pm 19.06^{*}$ & 6.20 \\
\hline
\end{tabular}

Datos presentados como media \pm desviación estándar $(n=9) . *=0.05), * *=p<0.01$

Cuadro 3. Volumen y características espermáticas en Piaractus brachypomus (media \pm desviación estándar)

\begin{tabular}{cccl}
\hline $\begin{array}{c}\text { Volumen } \\
\text { seminal }(\mathrm{ml})\end{array}$ & $\begin{array}{c}\text { Movilidad } \\
\text { espermática (\%) }\end{array}$ & $\begin{array}{c}\text { Concentración } \\
\text { espermática } \\
\left(\mathrm{spz} . \mathrm{x} 10^{6} / \mu \mathrm{l}\right)\end{array}$ & Autor \\
\hline $0.3 \pm 0.2$ & $91.2 \pm 4.3$ & $30 \pm 0.6$ & Fresneda et al. $(2004)^{1}$ \\
$13.4 \pm 1.5$ & $92 \pm 1$ & $17.7 \pm 1.8$ & Navarro et al. $(2004)$ \\
$1.8 \pm 0.6$ & $91.0 \pm 5.6$ & $55.5 \pm 2.3^{1}$ & Nascimento et al. $(2010)^{1}$ \\
$13 \pm 1$ & $86 \pm 1$ & $11 \pm 1$ & Ramírez-Merlano et al. $(2011 \mathrm{~b})$ \\
$7.44 \pm 2.18$ & $88.56 \pm 2.24$ & $18.58 \pm 4.90$ & Presente estudio $\left(1^{\text {ra }}\right.$ colecta) \\
$5.94 \pm 1.86$ & $87.44 \pm 3.54$ & $15.81 \pm 5.26$ & Presente estudio $\left(2^{\text {da }}\right.$ colecta $)$ \\
\hline
\end{tabular}

${ }^{1}$ Sin inducción hormonal

(2016), quienes reportaron intervalos de 35 días en el pacú Piaractus mesopotamicus obteniendo valores de VS similares entre colectas. Contrariamente, la frecuencia de espermiación (cada 1, 2 o 4 semanas) resultó en menores valores de VS, CE y espermatocrito, TA reducidos y ME menos vigorosa en trucha arco iris Salmo gairdneri (Büyükhatipoglu y Holtz, 1984).
La movilidad espermática presentó rangos similares a los reportados por otros autores (Fresneda et al., 2004; Navarro et al., 2004; Nascimento et al., 2010; RamírezMerlano et al., 2011 a) para P. brachypomus. La concentración espermática fue semejante a la descrita por Navarro et al. (2004), aunque menor a la obtenida por Fresneda et al. (2004) y Nascimento et al. (2010). Así 
Cuadro 4. Características del plasma seminal en algunas especies ícticas de la acuicultura colombiana

\begin{tabular}{|c|c|c|c|c|c|}
\hline Especie & $\mathrm{pH}$ & $\begin{array}{c}\text { Osm } \\
(\mathrm{mOsm} / \mathrm{kg})\end{array}$ & $\begin{array}{c}\mathrm{K}^{+} \\
(\mathrm{mmol} / \mathrm{l})\end{array}$ & $\begin{array}{c}\mathrm{Na}^{+} \\
(\mathrm{mmol} / \mathrm{l})\end{array}$ & Referencia \\
\hline $\begin{array}{l}\text { Trucha arcoíris } \\
\text { (Oncorhynchus } \\
\text { mykiss) }\end{array}$ & $8.1 \pm 0.2$ & $322.1 \pm 21.8$ & $30.4 \pm 4.5$ & $122.2 \pm 1$ & $\begin{array}{l}\text { Lahnsteiner et al. } \\
\text { (1998); Glogowski } \\
\text { et al. (2000) }\end{array}$ \\
\hline $\begin{array}{l}\text { Yamú } \\
\text { (Brycon }\end{array}$ & --- & $314.1 \pm 5.6$ & $22 \pm 0.7$ & $86 \pm 2.1$ & $\begin{array}{l}\text { Cruz-Casallas et } \\
\text { al. (2004) }\end{array}$ \\
\hline amazonicus) & $8.2 \pm 0.2$ & $273.5 \pm 15.3$ & $28.2 \pm 4.6$ & --- & $\begin{array}{l}\text { Jiménez-Molina et } \\
\text { al. (2016) }\end{array}$ \\
\hline $\begin{array}{l}\text { Bagre rayado } \\
\text { (Pseudoplatystoma } \\
\text { metaense) }\end{array}$ & --- & $259.9 \pm 10.6$ & $4.5 \pm 1$ & $144 \pm 7.7$ & $\begin{array}{l}\text { Ramírez-Merlano } \\
\text { et al. (2011a) }\end{array}$ \\
\hline $\begin{array}{l}\text { Sabaleta (Brycon } \\
\text { henni) }\end{array}$ & $7.5 \pm 0.5$ & $299.9 \pm 5.2$ & 27.8 & 255 & $\begin{array}{l}\text { Tabares (2005); } \\
\text { Tabares et al. } \\
(2006)\end{array}$ \\
\hline \multirow[t]{3}{*}{$\begin{array}{l}\text { Cachama blanca } \\
\text { (Piaractus } \\
\text { brachypomus) }\end{array}$} & $9.0 \pm 0.3$ & $313.3 \pm 14.0$ & --- & --- & $\begin{array}{l}\text { Nascimento et al. } \\
\text { (2010); Sampaio } \\
\text { Leite } \text { et al. (2014) }\end{array}$ \\
\hline & $8.1 \pm 0.3^{+}$ & $261.5 \pm 19.9^{+}$ & $38.1 \pm 4.7^{+}$ & $129.9 \pm 3.6^{+}$ & Presente estudio \\
\hline & $8.0 \pm 0.2^{++}$ & $307.6 \pm 19.1^{++}$ & $37.2 \pm 5.3^{++}$ & $134.8+3.5^{++}$ & \\
\hline
\end{tabular}

+ Presente estudio, primera colecta; ${ }^{++}$Presente estudio, segunda colecta

mismo, el volumen seminal fue inferior al de reproductores inducidos hormonalmente con EHC (Navarro et al., 2004; Ramírez-Merlano et al., 2011a), pero superior al de ejemplares no inducidos (Fresneda et al., 2004; Nascimento et al., 2010) (Cuadro 3).

La aplicación de EHC puede aumentar el VS como resultado de una mayor producción de fluido por parte de los túbulos seminíferos (Viveiros et al., 2002) y, en consecuencia, puede reducir la CE hasta en un $50 \%$ debido a un efecto diluyente (Maria et al., 2012). Además del efecto hormonal, otros factores como la temperatura, el fotoperiodo y la nutrición pueden afectar el VS, mientras que la ME puede ser afectada por la edad del reproductor, la osmolaridad y la concentración iónica del medio de fertilización (Fauvel et al., 2010).
En el aspecto fisicoquímico (Cuadro 2), la osmolaridad y la concentración de iones $\mathrm{Na}^{+}, \mathrm{Ca}^{2+}$ y TG aumentaron en la segunda colecta en contraposición a lo reportado por Munkittrick y Moccia (1987) y Hajirezaee et al. (2009), quienes reportaron reducción del $\mathrm{K}^{+}, \mathrm{Na}^{+}$y Cl- en Salmo gairdneri, así como descenso en iones mono y divalentes de la trucha café del caspio Salmo trutta caspius, estando en ambos casos asociado a la frecuencia de colecta y a la progresión de la temporada reproductiva.

El aumento en la osmolaridad seminal de $P$. brachypomus fue concomitante con el aumento de los iones $\mathrm{Na}^{+}, \mathrm{Ca}^{2+}$ y TG y, posiblemente, se encuentra asociado a cambios en la capacidad de los ductos espermáticos para proporcionar una hidratación seminal adecuada, a pesar del estímulo hormonal con 
EHC. En este sentido, variaciones en la composición plasmática seminal de salmónidos durante la temporada reproductiva ha sido ligada a cambios histomorfológicos en el epitelio germinativo testicular, ductos testiculares principales y ductos espermáticos, que son responsables de generar componentes minerales y orgánicos del semen (Lahnsteiner et al., 1993) en ciprínidos (Lahnsteiner et al., 1994) y blénidos (Lahnsteiner y Patzner, 1990; Lahnsteiner et al., 1990).

La osmolaridad plasmática de $P$. brachypomus se ubicó dentro de los rangos reportados por Alavi y Cosson (2006) para especies de agua dulce ( 230 y $360 \mathrm{mOsm} / \mathrm{kg}$ ). La concentración de $\mathrm{Na}^{+}$fue semejante al reportado para O. mykiss (Lahnsteiner et al., 1998; Glogowski et al., 2000) y P. metaense (Ramírez-Merlano et al., 2011b), mientras que $\mathrm{Ca}^{2+}$ fue superior a $B$. henni (Tabares, 2005; Tabares et al., 2007). El K ${ }^{+}$plasmático en $P$. brachypomus se encontró dentro del rango reportado por Ciereszko et al. (2011) para especies de agua dulce y supera las registradas en otras especies nativas, como $B$. amazonicus (Cruz-Casallas et al., 2004, 2007; Jiménez-Molina et al., 2016), P. metaense (Ramírez-Merlano et al., 2011b) y B. henni (Tabares et al., 2006). El Cl-, por otro lado, estuvo en el rango del $P$. metaense (Ramírez-Merlano et al., 2011b), por encima del B. amazonicus (Cruz-Casallas et al., 2004, Jiménez-Molina et al., 2016) y debajo del reportado por Sampaio-Leite et al. (2014) para la misma especie (Cuadro 4).

En este estudio, la concentración del $\mathrm{Mg}^{2+}$ fue inferior a la observada en $B$. amazonicus y $B$. henni (Cruz-Casallas et al., 2004; Tabares, 2005; Tabares et al., 2006), mientras que el $\mathrm{Ca}^{2+}$ fue superior con relación a otras especies (Cuadro 5). Los iones $\mathrm{Ca}^{2+} \mathrm{y} \mathrm{Mg}^{2+}$ son fundamentales para activar la ME en ciprínidos y salmónidos. En ciprínidos, la hiposmolaridad permite el ingreso del agua a través de acuaporinas de la membrana (Dzyuba y Cosson, 2014), el cambio de polaridad de la membrana inicia el movimiento flagelar del espermatozoide por reducción de concentraciones internas de $\mathrm{Na}^{+}, \mathrm{K}^{+}$, y el incremento de $\mathrm{Ca}^{2+}$ intracelular (Krasznai et al., 2003; Dumorné et al., 2017). En la carpa común Cyprinus carpio, el $\mathrm{Na}^{+}$ se intercambia con $\mathrm{Ca}^{2+}$ para iniciar la movilidad (Krasznai et al., 2003). En salmónidos, el ambiente acuático (con bajos niveles de $\mathrm{K}^{+}$) induce la salida de $\mathrm{K}^{+}$desde el interior celular, estimulando el ingreso de $\mathrm{Ca}^{2+} \mathrm{y} \mathrm{Mg}^{2+}$, que hiperpolarizan la membrana espermática y conducen a la activación de la movilidad (Morisawa, 2008).

En cuanto a la parte orgánica del plasma seminal, la concentración de TG se encuentra por encima de la reportada en Salmo trutta magrostigma (Bozkurt et al., 2011), Cyprinus carpio (Bozkurt et al., 2009), Ctenopharyngodon idella (Bozkurt et al., 2008), pero inferior a la reportada en Salmo trutta caspius (Hajirezaee et al., 2009), como se puede apreciar en el Cuadro 6.

En el presente estudio, los valores de glucosa fueron estadísticamente similares entre las dos colectas y se asemejaron a los reportados por Sampaio-Leite et al. (2014) para esta especie. Según Soengas et al. (1993), la glucosa del plasma seminal se relaciona con la demanda energética de los testículos durante la espermatogénesis o con la síntesis lipídica durante la producción de los espermatozoides. De esta manera, lípidos, glucosa y otros monosacáridos secretados hacia el fluido seminal son fuente primaria de energía durante el almacenamiento inmóvil de los espermatozoides en el testículo y durante la fase regenerativa del ciclo testicular (Lahnsteiner y Patzner, 2009). En los ductos testiculares y espermáticos se producen enzimas como la sintasa de ácidos grasos, glucosa 6-fosfatasa y glucosa 6-fosfato deshidrogenasa facilitando la producción de TG, así como la gluconeogénesis (Lahnsteiner et al., 1993). La presencia de estas enzimas permite presumir la existencia de una interacción bioquímica entre sustratos energéticos para la formación y mantenimiento de las células espermáticas. 
Cuadro 5. Concentración de iones $\mathrm{Ca}, \mathrm{Mg}$ y $\mathrm{Cl}$ en el plasma seminal de algunas especies ícticas de la acuicultura colombiana

\begin{tabular}{|c|c|c|c|c|}
\hline Especie & $\begin{array}{c}\mathrm{Ca}^{2+} \\
(\mathrm{mmol} / \mathrm{l})\end{array}$ & $\begin{array}{c}\mathrm{Mg}^{2+} \\
(\mathrm{mmol} / \mathrm{l})\end{array}$ & $\begin{array}{c}\mathrm{Cl}^{-} \\
(\mathrm{mmol} / \mathrm{l})\end{array}$ & Referencia \\
\hline $\begin{array}{l}\text { Trucha arcoíris } \\
\text { (Oncorhynchus } \\
\text { mykiss) }\end{array}$ & $1.1 \pm 0.3$ & $0.9 \pm 0.1$ & --- & $\begin{array}{l}\text { Lahnsteiner et al. } \\
\text { (1998); Glogowski et } \\
\text { al. (2000) }\end{array}$ \\
\hline $\begin{array}{l}\text { Yamú } \\
\text { (Brycon }\end{array}$ & --- & $8.4 \pm 0.4$ & --- & $\begin{array}{l}\text { Cruz-Casallas et al. } \\
(2004)\end{array}$ \\
\hline amazonicus) & --- & $0.6 \pm 0.2$ & $75.8 \pm 0.4$ & $\begin{array}{l}\text { Jiménez-Molina et al. } \\
\text { (2016) }\end{array}$ \\
\hline $\begin{array}{l}\text { Bagre rayado } \\
\text { (Pseudoplatystoma } \\
\text { metaense) }\end{array}$ & --- & $0.3 \pm 0.1$ & $118.2 \pm 5.3$ & $\begin{array}{l}\text { Ramírez-Merlano et } \\
\text { al. (2011a) }\end{array}$ \\
\hline $\begin{array}{l}\text { Sabaleta (Brycon } \\
\text { henni) }\end{array}$ & 2.03 & 5.1 & --- & $\begin{array}{l}\text { Tabares (2005); } \\
\text { Tabares et al. (2006) }\end{array}$ \\
\hline \multirow[t]{3}{*}{$\begin{array}{l}\text { Cachama blanca } \\
\text { (Piaractus } \\
\text { brachypomus) }\end{array}$} & --- & --- & $376.4 \pm 66.0$ & $\begin{array}{l}\text { Nascimento et al. } \\
\text { (2010); Sampaio Leite } \\
\text { et al. (2014) }\end{array}$ \\
\hline & $3.5 \pm 1.3+$ & $0.9 \pm 0.1^{+}$ & $119 \pm 8^{+}$ & Presente estudio \\
\hline & $11.9 \pm 0.2^{++}$ & $1.0 \pm 0.2^{++}$ & $125.4 \pm 3.4^{++}$ & \\
\hline
\end{tabular}

+ Primera colecta; ${ }^{++}$Segunda colecta

Cuadro 6. Composición orgánica del plasma seminal en especies ícticas de la acuicultura comercial

\begin{tabular}{lcccl}
\hline Especie & $\begin{array}{c}\text { Proteína } \\
(\mathrm{mg} / \mathrm{dl})\end{array}$ & $\begin{array}{c}\text { Triglicéridos } \\
(\mathrm{mg} / \mathrm{dl})\end{array}$ & $\begin{array}{c}\text { Glucosa } \\
(\mathrm{mg} / \mathrm{dl})\end{array}$ & Referencia \\
\hline $\begin{array}{l}\text { Trucha arcoiris } \\
\text { (Oncorhynchus mykiss })\end{array}$ & $124.1 \pm 30.8$ & --- & - & $\begin{array}{l}\text { Glogowski } \text { et } \\
\text { al. }(2000)\end{array}$ \\
$\begin{array}{l}\text { Trucha café (Salmo } \\
\text { trutta m. })\end{array}$ & $480 \pm 20$ & $6.24 \pm 0.08$ & --- & $\begin{array}{l}\text { Bozkurt } \text { et al. } \\
(2011)\end{array}$ \\
$\begin{array}{l}\text { Trucha café caspio } \\
\text { (Salmo trutta c. })\end{array}$ & $60 \pm 10$ & $26.3 \pm 17.5$ & $36.03 \pm 18$ & $\begin{array}{l}\text { Hajirezaee } \text { et } \\
\text { al. }(2009)\end{array}$ \\
$\begin{array}{l}\text { Carpa espejo } \\
\text { (Cyprinus carpio })\end{array}$ & $140 \pm 2$ & $10.3 \pm 1.0$ & --- & $\begin{array}{l}\text { Bozkurt } \text { et al. } \\
(2009)\end{array}$ \\
$\begin{array}{l}\text { Carpa herbívora } \\
\text { (Ctenopharyngodon }\end{array}$ & $370 \pm 80$ & $14.85 \pm 1.5$ & $1.36 \pm 0.11$ & $\begin{array}{l}\text { Bozkurt } \text { et al. } \\
\text { (2008) }\end{array}$ \\
$\begin{array}{l}\text { idella) } \\
\text { Cachama blanca } \\
\text { (Piaractus } \\
\text { brachypomus) }\end{array}$ & --- & $25.7 \pm 10.4$ & $20.3 \pm 14.1$ & $\begin{array}{l}\text { Sampaio-Leite } \\
\text { et al. } \text { (2014) }\end{array}$ \\
& $30.6 \pm 14.2^{+}$ & $17.56 \pm 2.9^{+}$ & $20.11 \pm 13.5^{+}$ & $\begin{array}{l}\text { Presente } \\
\text { estudio }\end{array}$ \\
\hline
\end{tabular}

${ }^{+}$Primera colecta; ${ }^{++}$Segunda colecta 
La concentración proteica de $P$. brachypomus fue inferior con respecto a otras especies (Cuadro 6). Loir et al. (1990) y Aramli et al. (2014) reportaron rangos entre 100 y $300 \mathrm{mg} / \mathrm{dl}$ en el plasma seminal de salmones y esturiones, respectivamente, corroborando la baja concentración de proteínas presentes en el semen de P. brachypomus de este estudio.

\section{Conclusiones}

- Colectas de semen en Piaractus brachypomus con un intervalo de 30 días permiten obtener semen de calidad similar de un mismo reproductor, sin variaciones significativas en los parámetros espermáticos, aunque pueden presentarse variaciones en la composición del plasma seminal.

- Las relaciones entre componentes plasmáticos son inconstantes y pueden estar afectados por el muestreo.

\section{Literatura Citada}

1. Alavi SM, Cosson J. 2005. Sperm motility in fishes. I. Effects of temperature and $\mathrm{pH}$ : a review. Cell Biol Int 29: 101-110. doi: 10.1016/ j.cellbi.2004.11.021

2. Alavi SM, Cosson J. 2006. Sperm motility in fishes. II. Effects of ions and osmolality: a review. Cell Biol Int 30: 114. doi: 10.1016/j.cellbi.2005.06.004

3. Alavi SM, Rodina M, Policar T, Linhart O. 2009. Relationship between semen characteristics and body size in Barbus barbus L. (Teleostei: Cyprinidae) and effects of ions and osmolality on sperm motility. Comp Biochem Phys A 153: 430-437 doi: 10.1016/ j.cbpa.2009.04.001

4. Aramli M, Kalbassi M, Nazari, R. 2014. Protein concentration and enzyme activities of fresh and frozen-thawed
Persian sturgeon, Acipenser persicus (Borodin, 1897) semen. Int Aquat Res 6: 54. doi: 10.1007/s40071-014-0054-x

5. Batlouni SR, Romagosa E, Borella MI. 2006. The reproductive cycle of male catfish Pseudoplatystoma fasciatum (Teleostei, Pimelodidae) revealed by changes of the germinal epithelium an approach addressed to aquaculture. Anim Reprod Sci 96: 116-132. doi: 10.1016/ j.anireprosci.2005.11.012

6. Bobe J, Labbé C. 2010. Egg and sperm quality in fish. Gen Comp Endocr 165: 535-548. doi: 10.1016/j.ygcen.2009.02.011

7. Bozkurt Y, Öðretmen F, Erçin U, Yéldéz U. 2008. Seminal plasma composition and its relationship with physical spermatological parameters of grass carp Ctenopharyngodon idella semen: with emphasis on sperm motility. Aquac Res 39: 1666-1672. doi: 10.1111/ j.1365-2109.2008.02041.x

8. Bozkurt Y, Ogretmen F, Secer S, Ercin U. 2009. Effects of seminal plasma composition on sperm motility in mirror carp (Cyprinus carpio). Isr $\mathrm{J}$ Aquacult-Bamid 61: 307-314.

9. Bozkurt Y, Ogretmen F, Kokcu O, Ercin U. 2011. Relationships between seminal plasma composition and sperm quality parameters of the Salmo trutta macrostigma (Dumeril, 1858) semen: with emphasis on sperm motility. Czech J Anim Sci 56: 355-364. doi: 10.17221/ 2394-CJAS

10. Büyükhatipoglu S, Holtz W. 1984. Sperm output in rainbow trout (Salmo gairdneri). Effect of age, timing and frequency of stripping and presence of females. Aquaculture 37: 63-71. doi: 10.1016/0044-8486(84)90044-9

11. Cejko BI, Krejszeff S, Sarosiek B, -arski D, Judycka S, Kowalski RK. 2015. Biochemical factors of common carp (Cyprinus carpio L). 1758, seminal plasma and its relationship with sperm motility parameters. J Appl Ichthyol 31: 10-17. doi: 10.1111/jai.12721 
12. Ciereszko A, Glogowski J, Dabrowski K. 2011. Biochemical characteristics of seminal plasma and spermatozoa of freshwater fishes. In: Tiersch TR, Green CC (eds). Cryopreservation in aquatic species. Louisiana, USA: World Aquaculture Society. p 46-79.

13. Cruz-Casallas PE, VelascoSantamaría YM, Ramírez-Ordoñez MP, Medina-Robles VM, Olivera-Angel M. 2004. Determinación de algunas características bioquímicas del plasma seminal del yamú Brycon siebenthalae. En: II Congreso Colombiano de Acuicultura.

14. Cruz-Casallas PE, Medina-Robles VM, Velasco-Santamaria YM. 2006. Determinación del espermatocrito y efecto del volumen de la dosis seminante sobre la fertilidad en yamú Brycon amazonicus. Rev Colomb Cienc Pec 19: 140-145.

15. Cruz-Casallas PE, Medina-Robles VM, Velasco-Santamaría YM. 2007. Seasonal variation of sperm quality and the relationship between spermatocrit and sperm concentration in Yamú Brycon amazonicus. N Am J Aquacult 69: 159165. doi: 10.1577/A06-002.1

16. Cruz-Casallas PE, Medina-Robles VM, Velasco-Santamaría YM. 2011. Fish farming of native species in Colombia: current situation and perspectives. Aquac Res 42: 823-831. doi: 10.1111/ j.1365-2109.2011.02855.x

17. De Souza TG, Hainfellner $P$, Kuradomi RY, Muñoz ME, Honji RM, Moreira RG, Batlouni SR. 2015. Inappropriate management conditions, especially for the regressed class, are related to sperm quality in Prochilodus lineatus. Theriogenology 83: 797-807. doi: 10.1016/j.theriogenology.2014.11.018

18. Dumorné K, Figueroa E, Cosson J, Lee Estévez M, Ulloa Rodríguez, $P$, Valdebenito I, Farías JG 2017. Protein phosphorylation and ions effects on salmonid sperm motility activation. Rev Aquacult 10: 727-737. doi: 10.1111/ raq. 12198
19. Dzyuba V, Cosson J. 2014. Motility of fish spermatozoa: from external signaling to flagella response. Reprod Biol 14: 165175. doi: 10.1016/j.repbio.2013.12.005

20. Fauvel C, Suquet M, Cosson J. 2010. Evaluation of fish sperm quality. J Appl Ichthyol 26: 636-643. doi: 10.1111/j.14390426.2010.01529.x

21. Fresneda A, Lenis G, Agudelo E, Olivera-Ángel M. 2004. Espermiación inducida y crioconservación de semen de Cachama. Rev Colomb Cienc Pec 17: 46-52.

22. Glogowski J, Kwasnik M, Piros B, Dabrowski K, Goryczko K, Dobosz S, Kuzminski H, et al. 2000. Characterization of rainbow trout milt collected with a catheter: semen parameters and cryopreservation successes. Aquac Res 31: 289-296. doi: 10.1046/j.13652109.2000.00400.x

23. Hajirezaee S, Amiri BM, Mirvaghefi $A R$. 2009. Effects of stripping frequency on semen quality of endangered Caspian brown trout, Salmo trutta caspius. Am J Anim Vet Sci 4: 65-71. doi: 10.3844/ ajavsp.2009.65.71

24. Hickman CG. 1958. Spermatocrit values in facilitating the estimation of spermatozoa concentrations. J Dairy Sci 41: 318-319. doi: 10.3168/jds.S00220302(58)90914-7

25. Jiménez-Molina WR, Suárez-Martínez RO, Medina-Robles VM, CruzCasallas PE. 2016. Evaluación de la calidad seminal en yamú Brycon amazonicus: relación con la viabilidad posdescongelación. En: XXII Jornada de Acuicultura - IALL. Villavicencio, Colombia.

26. Krasznai Z, Morisawa M, Morisawa S, Krasznai ZT, Trón L, Gáspár R, Márián T. 2003. Role of ion channels and membrane potential in the initiation of carp sperm motility. Aquat Living Resour 16: 445-449. doi: 10.1016/S09907440(03)00054-8

27. Kuradomi RY, De Souza TG, Foresti F, Schulz RW, Bogerd J, Moreira RG, Furlan LR, et al. 2016. Effects of re- 
stripping on the seminal characteristics of pacu (Piaractus mesopotamicus) during the breeding season. Gen Comp Endocr 225: 162-173. doi: 10.1016/ j.ygcen.2015.06.007

28. Lahnsteiner F, Richtarski U, Patzner $R A$. 1990. Functions of the testicular gland in two blenniid fishes, Salaria (=Blennius) pavo and Lipophrys (= Blennius) dalmatinus (Blenniidae, Teleostei) as revealed by electron microscopy and enzyme histochemistry. J Fish Biol 37: 85-97. doi: 10.1111/j.10958649.1990.tb05930.x

29. Lahnsteiner F, Patzner RA. 1990. The spermatic duct of blenniid fish (Teleostei, Blenniidae) fine structure, histochemistry and function. Zoomorphology 110: 63-73. doi: 10.1007/BF01632813

30. Lahnsteiner F, Patzner RA, Weismann T. 1993. Energy resources of spermatozoa of the rainbow trout Oncorhynchus mykiss (Pisces, Teleostei). Reprod Nutr Dev 33: 349-360. doi: 10.1051/ rnd:19930404

31. Lahnsteiner F, Patzner RA, Weismann T. 1994. Testicular main ducts and spermatic ducts in some cyprinid fishes I. Morphology, fine structure and histochemistry. J Fish Biol 44: 937-951. doi: 10.1111/j.1095-8649.1994.tb01266.x

32. Lahnsteiner F, Berger B, Weismann T, Patzner RA. 1998. Determination of semen quality of the rainbow trout, Oncorhynchus mykiss, by sperm motility, seminal plasma parameters, and spermatozoal metabolism. Aquaculture 163: 163-181. doi: 10.1016/S00448486(98)00243-9

33. Lahnsteiner F, Patzner RA. 2009. Male reproductive system: spermatic duct and accessory organs of the testis. In: Jamieson BGM (ed). Reproductive biology and phylogeny of fishes (Agnathans and bony fishes). New Hampshire, USA: Science Publishers. $p$ 143-186.

34. Loir M, Labbé C, Maisse G, Pinson A, Boulard G, Mourot B, Chambeyron F. 1990. Proteins of seminal fluid and spermatozoa in the trout Oncorhynchus mykiss: partial characterization and variations. Fish Physiol Biochem 8: 485495. doi: 10.1007/BF00003405

35. Maria AN, Azevedo HC, Santos JP, Carneiro PC. 2012. Hormonal induction and semen characteristics of tambaqui Colossoma macropomum. Zygote 20: 39-43. doi: 10.1017/S0967199410000559

36. Migaud H, Bell G, Cabrita E, McAndrew B, Davie A, Bobe J, Herráez MP, et al. 2013. Gamete quality and broodstock management in temperate fish. Rev Aquacult 5: 194-223. doi: 10.1111/raq.12025

37. [MADR] Ministerio de Agricultura y Desarrollo Rural. 2018. Dirección de Cadenas Pecuarias, Pesqueras y Acuícolas. Cadena de la Acuicultura. [Internet]. Disponible en: https://sioc.minagricultura.gov.co/Acuicultura/Boletines/

38. Mochida K, Kondo T, Matsubara T, Adachi S, Yamauchi K. 1999. A high molecular weight glycoprotein in seminal plasma is a sperm immobilizing factor in the teleost Nile tilapia, Oreochromis niloticus. Dev Growth Differ 41: 619627. doi: 10.1046/j.1440-169x.1999.00463.x

39. Morisawa M, Suzuki K, Morisawa S. 1983a. Effects of potassium and osmolality on spermatozoan motility of salmonid fishes. J Exp Biol 107: 105-113.

40. Morisawa M, Suzuki K, Shimizu H, Morisawa S, Yasuda K. 1983b. Effects of osmolality and potassium on motility of spermatozoa from freshwater cyprinid fishes. J Exp Biol 107: 95-103.

41. Morisawa M. 2008. Adaptation and strategy for fertilization in the sperm of teleost fish. J Appl Ichthyol 24: 362-370. doi: 10.1111/j.1439-0426.2008.01126.x

42. Munkittrick KR, Moccia RD. 1987. Seasonal changes in the quality of rainbow trout Salmo gairdneri semen: effect of a delay in stripping on spermatocrit, motility, volume and seminal plasma constituents. Aquaculture 64: 147-156. doi: 10.1016/0044-8486(87)-90350-4 
43. [NRC] National Research Council. 2011. Guide for the care and use of laboratory animals. $8^{\text {th }}$ ed. Washington, USA: NRC. 220 p. [Internet]. Available in: https://grants.nih.gov/grants/olaw/ guide-for-the-care-and-use-oflaboratory-animals.pdf

44. Nascimento AF, Maria AN, Pessoa NO, Carvalho MA, Viveiros AT. 2010. Out-of-season sperm cryopreserved in different media of the Amazonian freshwater fish pirapitinga Piaractus brachypomus. Anim Reprod Sci 118: 324-329. doi: 10.1016/j.anireprosci. 2009.07.002

45. Navarro O, Velasco-Santamaría YM, Cruz-Casallas PE. 2004. Evaluación de cinco protectores para la crioconservación de semen de cachama blanca Piaractus brachypomus. Rev Colomb Cienc Pec 17: 53-59.

46. Ramírez-Merlano JA, VelascoSantamaría YM, Medina-Robles VM, Cruz-Casallas PE. 2011a. Cryopreservation effects on the sperm quality of cachama blanca Piaractus brachypomus (Cuvier 1818). Aquac Res 42: 738745. doi: $10.1111 / \mathrm{j} .1365-2109.2011$.02835. $\mathrm{x}$

47. Ramírez-Merlano JA, Medina-Robles VM, Cruz-Casallas PE. 2011b. Variación estacional de las características seminales del bagre rayado Pseudoplatystoma metaense (Telostei, pimelodidae). Rev MVZ Cordoba 16: 2336-2348.

48. Rashid I, Hossain MS, Salam MA, Rafiquzzaman SM. 2018. Evaluation of seminal plasma composition and spermatozoa quality parameters of silver barb, Barbonymus gonionotus Bleeker, 1850. Fish Physiol Biochem 45: 105-114. doi: 10.1007/s10695-018-0539-4

49. Robles V, Herráez P, Cabrita E. 2008. Sperm quality assesment. In: Cabrita $E$, Herráez P (eds). Methods in reproductive aquaculture. Marine and Freshwater Species. CRC Press. p 93-147.
50. Rurangwa E, Kime DE, Ollevier F, Nash JP. 2004. The measurement of sperm motility and factors affecting sperm quality in cultured fish. Aquaculture 234: 1-28. doi: 10.1016/ j.aquaculture.2003.12.006

51. Sampaio Leite J, Melo-Maciel MAP, Ribeiro Pinheiro RR, Silva Pinheiro JP, Salmito-Vanderley CSB, Ferreira Nunes J. 2014. Avaliacao da presenca de glicose, cloreto e triglicerídeo no plasma seminal de tambaqui e pirapitinga. In: VII Congresso Norte-Nordeste de Reprodução Animal. Brasil.

52. Soengas JL, Sanmartín B, Barciela P, Aldegunde M, Rozas G. 1993. Changes in carbohydrate metabolism in domesticated rainbow trout (Oncorhynchus mykiss) related to spermatogenesis. Comp Biochem Phys B 105: 665671. doi: 10.1016/0305-0491(93)90103-C

53. Tabares CJ. 2005. Evaluación del efecto de algunos iones sobre la activación de la movilidad espermática y el potencial de membrana en Brycon henni (Eigenmann, 1913). Tesis de Magíster. Medellín, Colombia: Univ. de Antioquia. $68 \mathrm{p}$.

54. Tabares CJ, Montoya-López AF, Arboleda L, Echeverri A, Restrepo LF, Olivera-Ángel M. 2006. Efecto de la pluviosidad y el brillo solar sobre la producción y características del semen en el pez Brycon henni (Pisces Characidae). Rev Biol Trop 54: 179-187. doi: 10.15517/rbt.v54i1.13992

55. Tabares CJ, Ruíz T, Arboleda L, Olivera M. 2007. Effect of some ions on sperm activation in Brycon henni (Eligenmann, 1913). Acta Biol Colomb 12: 87-98.

56. Viveiros ATM, Fessehayey ter Veld M, Schulz RW, Komen J. 2002. Handstripping of semen and quality after maturational hormone treatments, in African catfish Clarias gariepinus. Aquaculture 213: 373-386. doi: 10.1016/ S0044-8486(02)00036-4 